\title{
Fertilización artificial aplicado a la conservación de la rana hojarasca desvanciente (Craugastor evanesco)
}

\author{
Samaniego, Diego \\ Instituto Smithsonian de Investigaciones Tropicales \\ Panamá, Panamá \\ samaniego.33@hotmail.com \\ Arcia, Igli \\ Instituto Smithsonian de Investigaciones Tropicales \\ Panamá, Panamá \\ arciaigli@hotmail.com \\ Della Togna, Gina \\ Instituto Smithsonian de Investigaciones Tropicales \\ Universidad Interamericana de Panamá \\ Panamá, Panamá \\ gina.dellatogna@uip.pa
}

\section{Abstract}

Within vertebrates, amphibians represent a highly diverse biological class facing the most critical situation. Difficulties exist within captive breeding programmes that limit the successful reproduction of these species and their subsequent reintroduction. The priority of establishing ex situ conservation programmes has led to the creation of captive conservation centres with the aim of keeping these species alive and reproducing genetically viable specimens. Among these is the lack of knowledge about the general biology and the specific reproductive characteristics of each species. Craugastor evanesco is one of our endemic species that lives in the Atlantic basin and Coclé, barely described in 2010 and is very susceptible to infection of the pathogenic fungus $B d$, with high probability of disappearing in the wild. Because of the above, our overall goal is to develop an artificial fertilization protocol applied to the conservation of the Vanishing Rainfrog (Craugastor evanesco). For this purpose, the stimulation effectiveness of a single ovulatory dose should be compared to the stimulation effectiveness using previous preparatory doses of lower concentration followed by the administration of the higher concentration ovulatory dose. Eggs obtained by stimulation with the most effective treatment (s) will be placed in a Petri dish moistened with ARS. High quality sperm samples (motility and progressive motion > 
$90 \%)$ shall be distributed over the egg mass using a pipette.

Keywords: Amphibian, artificial fertilization, eggs, hormones, reproduction.

\section{Resumen}

Dentro de los vertebrados, los anfibios representan una clase biológica de gran diversidad que enfrenta la situación más crítica. Existen dificultades dentro de los programas de reproducción en cautiverio que limitan la reproducción exitosa de dichas especies y su posterior reintroducción. La prioridad de establecer programas de conservación ex situ ha promovido la creación de centros de conservación en cautiverio con la finalidad de mantener vivas a estas especies y reproducir especímenes genéticamente viables Entre ellas, el poco conocimiento sobre la biología general y las características reproductivas específicas de cada especie. Craugastor evanesco es una de nuestras especies endémicas que habita en la cuenca atlántica y Coclé, apenas descrita en el año 2010 y es muy susceptible a la infección del hongo patógeno $B d$, con alta probabilidad de desaparecer en vida silvestre. Por lo mencionado anteriormente, nuestro objetivo general es desarrollar un protocolo de fertilización artificial aplicado a la conservación de la rana hojarasca desvaneciente (Craugastor evanesco). Para este fin se comparará la efectividad de estimulación de una sola dosis ovulatoria vs la efectividad de estimulación utilizando dosis preparatorias previas de menor concentración seguidas de la administración de la dosis ovulatoria de mayor concentración. Los huevos obtenidos mediante estimulación con el/los tratamientos más efectivos serán colocados en un plato Petri humedecido con ARS. Las muestras de esperma de alta calidad (motilidad y movimiento progresivo > 90\%) serán distribuidas sobre la masa de huevos utilizando una pipeta.

Palabras claves: anfibios, fertilización artificial, huevos, hormonas, reproducción.

\section{INTRODUCCIÓN}

La clase "vertebrados" está experimentando declives poblaciones y extinciones[1], [2]. De estos, los anfibios se sitúan en la posición más delicada, con un aproximado de 41 a $53 \%$ de especies amenazadas a nivel global[3].

Este panorama enmarca la llamada "Crisis de extinción de anfibios", ocasionando declives 
y extinciones de anuros, salamandras y cecilias distribuidos en diferentes ecosistemas de nuestro planeta, excepto en el continente antártico[4], [5].

Panamá se ubica en la región tropical mesoamericana, que alberga endemismo y diversidad, con un total de 241 especies descritas de anfibios[3], [6]-[9]. La especie Craugastor evanesco es de reproducción terrestre y desarrollo directo. Se distribuye en área de El Copé y la vertiente atlántica de la serranía de Tabasará[10]. Su estatus de conservación es de críticamente amenazada, por razones como la enfermedad causada por el hongo Batrachochytrium dendrobatidis[11], destrucción de hábitat para agricultura y actividades relacionadas a la explotación de recursos mineros[12].

La especie se encuentra dentro de Proyecto de Rescate y Conservación de Anfibios, un programa ex-situ de conservación y reproducción en cautiverio (CBP) [13]-[16].

Las tecnologías de reproducción asistida se aplican para ayudar a reproducir especies que no se reproducen fácilmente de manera natural en CBP's y así, evitar su extinción [17][19].

\section{MÉTODO}

\section{A. SELECCIÓN}

Se seleccionarán individuos sexualmente maduros.

\section{B. COLECTA DE ESPERMA}

Cuerpo del Texto: Se realizará tratamiento de estimulación hormonal para colecta de orina espermática, vía inyección intracelómica del agonista de la hormona liberadora de la gonadotropina GnRH-des-Gly10, D-Ala6, Pro-NHEt9.

Se analizarán los parámetros espermáticos generales (concentración, motilidad, movimiento progresivo, $\mathrm{pH}$, osmolalidad, morfología).

\section{COLECTA DE HUEVOS}

Se realizará tratamiento de estimulación hormonal vía inyección intracelómica del agonista de la hormona liberadora de la gonadotropina GnRH-des-Gly10, D-Ala6, ProNHEt9 y la gonadotropina coriónica humana (hCG).

Se probará la efectividad de las hormonas y las concentraciones para la colecta de huevos viables.

Se comparará la efectividad de estimulación una dosis ovulatoria (Fig. 1) vs la efectividad de dosis preparatorias previas de menor concentración seguidas de la dosis ovulatoria de mayor concentración (Fig. 2).

La dosis ovulatoria será administrada 72 horas después de la estimulación con la dosis preparatoria para cada uno de los tratamientos (Fig. 2). 


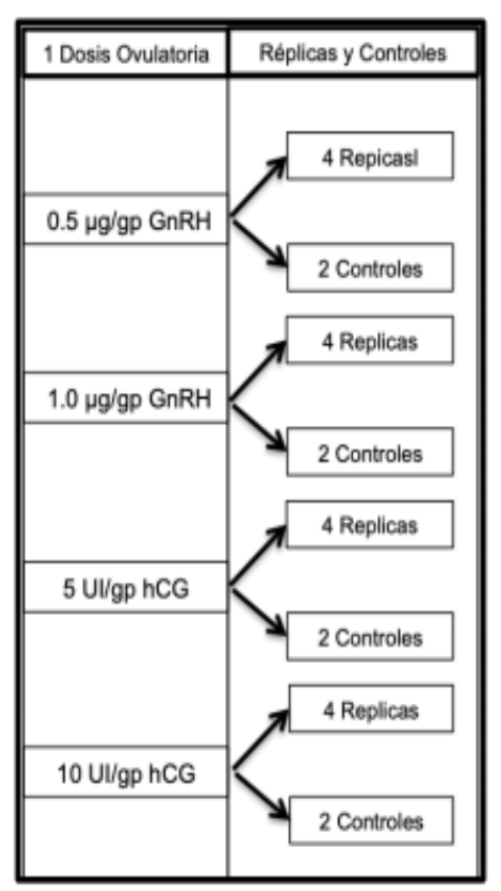

Figura 1: Experimento 1 con dosis ovulatorias

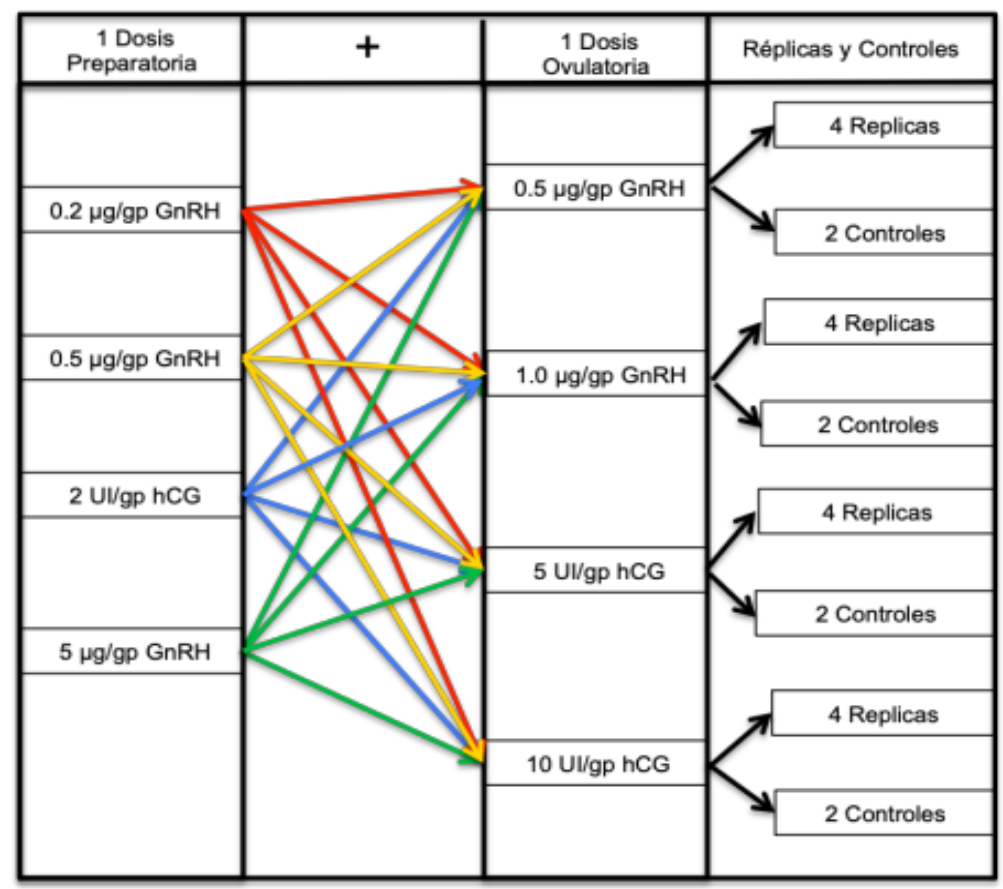

Figura 2: Experimento 2 con dosis preparatorias más dosis ovulatorias.

\section{MONITOREO Y EVALUACION DE HUEVOS}

Se regresarán a sus terrarios bajo constante observación. Si después de pasadas 24 horas de la estimulación, la hembra no oviposita naturalmente, se procederá a realizar masajes abdominales diarios por 5 días para estimular la oviposición.

Los huevos colectados serán colocados en platos Petri ligeramente humedecidos con ARS y protegidos de exposición a la luz. Se contará el número total de huevos por evento de oviposición, se medirá el diámetro de los huevos y se evaluará la integridad de la capa de gel.

\section{E. FERTILIZACIÓN ARTIFICIAL}

Se realizará la técnica de fertilización artificial.

Se seleccionarán los huevos obtenidos mediante estimulación con el/los tratamientos más efectivos y serán colocados en un plato Petri humedecido con ARS. Las muestras de esperma de alta calidad (motilidad y movimiento progresivo >90\%) serán distribuidas sobre la masa de huevos utilizando una pipeta. 


\section{RESULTADOS ESPERADOS}

\section{A. Estimulación hormonal}

- Primera identificación del efecto de la estimulación hormonal en la producción de huevos en $C$. evanesco.

- Comparación del efecto estimulatorio hormonal entre las diferentes hormonas y concentraciones para la colecta de huevos en C. evanesco.

\section{B. Caracterización de los huevos}

Primer análisis de las características de los huevos (cantidad, diámetro e integridad de capa de gel) de la especie en cautiverio C. evanesco.

\section{Protocolode fertilización artificial}

- Primer estudio para la obtención de huevos fertilizados de forma artificial para esta especie.

- Desarrollo de técnicas de reproducción asistida específica para C. evanesco.

\section{IMPACTOS}

Los resultados de esta propuesta generarán conocimiento, actualmente inexistente, que impactará directamente en el manejo de especies de anfibios en peligro de extinción que se encuentran en cautiverio, y a su vez, producirá nueva información sobre la fisiología reproductiva de estas especies en su medio natural.

\section{Referencias}

[1] R. Dirzo, H. S. Young, M. Galetti, G. Ceballos, N. J. B. Isaac, and B. Collen, "Defaunation in the Anthropocene," Science (80-. )., vol. 345, no. 6195, pp. 401-406, 2014, doi: 10.1126/science.1251817.

[2] G. Ceballos, A. García, and P. R. Ehrlich, "The sixth extinction crisis loss of animal populations and species," J. Cosmol., vol. 8, no. November 2009, pp. 1821-1831, 2010, doi: 10.1016/j.patbio.2007.08.005.

[3] IUCN, "The IUCN Red List of Threatened Species.," Version 2021-1, 2021. https://www.iucnredlist.org (accessed Jun. 07, 2021).

[4] K. C. Zippel and J. R. Mendelson III, "The amphibian extinction crisis: A call to action," Herpetol. Rev., vol. 39, no. 1, pp. 23-29, 2008.

[5] A. Catenazzi, "State of the World 's Amphibians," Annu. Rev. Environ. Resour., vol. 40, pp. 91-119, 2015, doi: 10.1146/annurev-environ-102014-021358.

[6] Autoridad Nacional del Ambiente, Plan de Acción para la Conservación de los Anfibios en Panamá. Panama, 2011, p. 28.

[7] K. R. Lips and M. A. Donnelly, "Lessons from the tropics," Amphib. Declines Conserv. Status United States Species, no. June 2005, pp. 198-205, 2005, doi: 10.1525/california/9780520235922.003.0028.

[8] K. R. Lips, P. A. Burrowes, J. R. Mendelson, and G. Parra-Olea, "Amphibian population declines in Latin America: A synthesis," Biotropica, vol. 37, no. 2, pp. 222-226, 2005, doi: 10.1111/j.1744-7429.2005.00029.x.

[9] S. M. Whitfield, K. R. Lips, and M. A. Donnelly, "Amphibian Decline and Conservation in Central America," Copeia, vol. 104, no. 2, pp. 351-379, 2016, doi: 10.1643/CH-15-300.

[10] M. J. Ryan, J. M. Savage, K. R. Lips, and J. T. Giermakowski, "A new species of the Craugastor rugulosus 
series (Anura: Craugastoridae) from west-central Panama," Copeia, no. 3, pp. 405-409, 2010, doi: 10.1643/ $\mathrm{CH}-09-154$.

[11] A. J. Crawford, K. R. Lips, and E. Bermingham, "Epidemic disease decimates amphibian abundance, species diversity, and evolutionary history in the highlands of central Panama," Proc. Natl. Acad. Sci. U. S. A., vol. 107, no. 31, pp. 13777-13782, 2010, doi: 10.1073/pnas.0914115107.

[12] IUCN SSC Amphibian Specialist Group, "Craugastor evanesco," The IUCN Red List of Threatened Species 2019: e.T50922873A50922883., 2019. .

[13] C. H. R. Lewis et al., "Conserving Panamanian harlequin frogs by integrating captive-breeding and research programs," Biol. Conserv., vol. 236, no. October 2018, pp. 180-187, 2019, doi: 10.1016/j.biocon.2019.05.029.

[14] B. Gratwicke and J. B. Murphy, "Amphibian Conservation Efforts at the Smithsonian 's National Zoological Park and Conservation Biology Institute," Herpetol. Rev., vol. 47, no. 4, pp. 336-337, 2016.

[15] B. Gratwicke et al., "Evaluating the probability of avoiding disease-related extinctions of Panamanian amphibians through captive breeding programs," Anim. Conserv., vol. 19, no. 4, pp. 324-336, 2015, doi: 10.1111/acv.12249.

[16] Qazi A. Hussain, "Global amphibian declines: A review," Int. J. Biodivers. Conserv., vol. 4, no. 10, pp. 348357, 2012, doi: 10.5897/ijbc12.008.

[17] R. K. Browne, K. Wolfram, G. García, M. F. Bagaturov, and Z. J. J. . M. Pereboom, "Zoo-based amphibian research and conservation breeding programs," Amphib. Reptil. Conserv., vol. 5, no. 3, pp. 1-14, 2011.

[18] J. Clulow, R. Upton, V. L. Trudeau, and S. Clulow, Eds., "Amphibian Assisted Reproductive Technologies: Moving from Technology to Application," in Reproductive Sciences in Animal Conservation, vol. 1200, 2019.

[19] G. Della Togna, L. G. Howell, J. Clulow, C. J. Langhorne, R. Marcec-Greaves, and N. E. Calatayud, "Evaluating amphibian biobanking and reproduction for captive breeding programs according to the Amphibian Conservation Action Plan objectives," Theriogenology, vol. 150, no. xxxx, pp. 412-431, 2020, doi: 10.1016/j. theriogenology.2020.02.024.

\section{Autorización y Licencia CC}

Los autores autorizan a APANAC XVIII a publicar el artículo en las actas de la conferencia en Acceso Abierto (Open Access) en diversos formatos digitales (PDF, HTML, EPUB) e integrarlos en diversas plataformas online como repositorios y bases de datos bajo la licencia CC:

Attribution-NonCommercial-ShareAlike 4.0 International (CC BY-NC-SA 4.0) https://creativecommons. org/licenses/by-nc-sa/4.0/.

Ni APANAC XVIII ni los editores son responsables ni del contenido ni de las implicaciones de lo expresado en el artículo. 